\title{
Late Impact of COVID-19 Pneumonia on Testosterone Levels in Recovered, Post-Hospitalized Male Patients
}

\author{
Mohamed M. Aboelnaga ${ }^{\mathrm{a}, \mathrm{d}}\left(\mathbb{0}\right.$, Ahmed Abdelrazek $^{\mathrm{b}}$, Nahed Abdullah $^{\mathrm{c}}$, Mostafa El Shaer $^{\mathrm{c}}$
}

\begin{abstract}
Background: Recent studies reported lower androgen levels in coronavirus disease 2019 (COVID-19) hospitalized patients which linked to a worse prognosis. However, these studies were conducted in patients with active disease and long-term consequences are still not yet known. Therefore, we aimed to evaluate the late impact of COVID-19 pneumonia on testosterone levels in late recovered, post-hospitalized males.

Methods: We enrolled 55 COVID-19 pneumonia-recovered, posthospitalized male patients after an average of 60 days of discharge from the hospital and 40 age, body mass index (BMI), and waistmatched participants enrolled in this case-control study. The total testosterone cut-off point was set as $300 \mathrm{ng} / \mathrm{dL}$ and the cut-off point for luteinizing hormone (LH) was set as $9.4 \mathrm{IU} / \mathrm{L}$.
\end{abstract}

Results: Testosterone deficiency prevalence was $50.9 \%$, and testosterone deficiency was mainly hypogonadotropic (60.7\%). Late recovered COVID-19 pneumonia patients had significantly lower total testosterone and calculated free testosterone and higher testosterone to $\mathrm{LH}$ ratio and prevalence of testosterone deficiency in comparison with the control group. Testosterone-deficient COVID-19 pneumonia patients had significantly higher anthropometric parameters such as waist circumference and BMI, higher Charlson Comorbidity Index, and longer hospitalization time in comparison with testosterone-sufficient patients. Visceral obesity estimated with waist circumference was a significant predictor for total testosterone levels in COVID patients in regression analysis.

Conclusion: Late recovered post-hospitalized COVID-19 pneumonia patients still have lower androgen levels and a higher prevalence of testosterone deficiency in comparison with matched control participants. Moreover, visceral obesity was the most significant predictor

Manuscript submitted May 10, 2021, accepted June 15, 2021

Published online July 25, 2021

aEndocrinology \& Diabetes Unit, Mansoura Faculty of Medicine, Egypt ${ }^{b}$ Tropical medicine department, Mansoura Faculty of Medicine, Egypt 'Specialized Medical Hospital, Mansoura University, Mansoura, Egypt ${ }^{\mathrm{d}}$ Corresponding Author: Mohamed M. Aboelnaga, Faculty of Medicine, Mansoura University, 2 El Gomhouria Street, Mansoura 35516, Egypt.

Email: dr.mhd.endocrine@gmail.com

doi: https://doi.org/10.14740/jem749 of low testosterone levels in recovered COVID-19 patients.

Keywords: COVID-19; Pneumonia; Testosterone; Male; Obesity

\section{Introduction}

On March 12, 2020, World Health Organization (WHO) declared coronavirus disease (COVID-19) a global pandemic. COVID-19 is caused by a previously unknown beta-coronavirus, which is now named severe acute respiratory syndrome coronavirus 2 (SARS-CoV-2) due to its high sequence similarity with SARS-CoV. At the time of finishing this work, it has infected more than 180 million people worldwide and caused nearly 4 million deaths globally. COVID-19 is a systemic disease, affecting the respiratory, cardiovascular, digestive, endocrine, and neurologic systems [1]. Hyper-inflammation and immunosuppression are the prominent features in COVID-19 [2].

Many research had shown that men are more likely to get worse symptoms with higher hospitalization and mortality rate compared to females [3]. Furthermore, sex differences in the prevalence of smoking, drinking habits, and heart diseases do not fully explain the differences between both sexes regarding SARS-CoV-2 infection [4]. Consequently, a potential role for testosterone was hypothesized.

On the other hand, studies have identified angiotensinconverting enzyme 2 (ACE2) as a host cell receptor that SARS$\mathrm{CoV}-2$ can use to establish an infection and consequently provide a critical link between immunity, inflammation, ACE2, and cardiovascular disease [5]. In the same consent, many studies reported that ACE2 is widely expressed in testicular tissue, Leydig cells, and Sertoli cells [6]. ACE2 is a constitutive product of adult-type Leydig cells and may participate in the control of testicular function [7].

Therefore, the impact of COVID-19 infection on the hypothalamic-pituitary-gonadal axis attracted attention. Recent studies reported lower androgen levels in COVID-19 hospitalized patients which linked to worse prognosis and even fatal outcomes $[8,9]$. However, these studies were conducted in patients with active disease and long-term consequences are still not yet known and consequently, the effects of COVID-19 on the male reproductive system are not clarified. So, recovered patients from COVID-19 pneumonia should be evaluated to 
detect the late impact of COVID-19 pneumonia on male reproductive hormones levels.

Therefore, in this study we aimed to evaluate the late impact of COVID-19 pneumonia on male reproductive hormones, total testosterone (TT), and luteinizing hormone (LH), in recovered, post-hospitalized males in comparison with matched participants as controls.

\section{Materials and Methods}

Fifty-five COVID-19 pneumonia-recovered, post-hospitalized male patients, aged $35-65$ years (mean age $52.1 \pm 7.41$ years) after recovery and discharge from governmental isolation hospitals and 40 matched participants according to age, body mass index (BMI) and waist circumference (WC) were recruited in this study from January 2021 to March 2021 in Mansoura General Hospital, Mansoura University, Egypt. The control group was selected from the outpatient clinics of other departments at Mansoura University General Hospital. Our control participant group was treated for other medical disorders. The control group was matched according age and anthropometric parameters including BMI and WC. Our Institutional Research Board at Mansoura Faculty of Medicine reviewed and approved the study protocol (IRB no. R.21.03.1261). The study was conducted in compliance with the Declaration of Helsinki.

Eligibility criteria were patients post-hospitalized and recovered from COVID-19 pneumonia, after discharge from hospital, aged between 18 and 65 years, and male sex. While exclusion criteria were female sex, abnormal albumin levels, acute or chronic liver cell failure, history of the gonadal disease, malignancy, and endocrinal disease affecting testosterone levels (including diabetes mellitus (DM), pituitary disorders particularly hyperprolactinemia, and abnormal thyroid functions), testosterone replacement, medications or diseases that affect testosterone levels such as gonadotropin therapy, and patients on corticosteroid therapy in the last 4 weeks. Hospitalization for COVID-19 pneumonia was indicated in patients with blood oxygen saturation $<90 \%$. Intensive care unit (ICU) admission was indicated for patients who need mechanical support for their respiration by either continuous positive airway pressure-bilevel positive airway pressure (CPAP-BPAP) or mechanical ventilation. We excluded mild COVID pneumonia case to ensure homogeneity of the patients. Most of hospitalized patients for COVID pneumonia received corticosteroid therapy in form of dexamethasone or methyl prednisolone for variable time tailored according to patients' status, yet, we tested patients at least 4 weeks after the end of corticosteroid therapy to avoid interaction between corticosteroid and testosterone levels.

All data of the participants were checked and reviewed. Each participant underwent a detailed history with special stress for disease that affected gonadal functions and history of drug intake, particularly corticosteroid, and clinical examination. Most of our patients in this study were still on anticoagulant therapy. The comorbidity burden was assessed by the Charlson Comorbidity Index (CCI) [10]. Blood pressure, weight, height, BMI (calculated by the standard formula: weight $(\mathrm{kg}) /$ height $\left.^{2}(\mathrm{~m})^{2}\right)$, and $\mathrm{WC}$ were done for all partici- pants. WC was taken as the smallest circumference around the abdomen without any clothing at the umbilical level.

\section{Laboratory evaluation}

All laboratory tests were performed after an average of 60 days of discharge from isolation governmental hospitals. The participants and recovered patients were tested in the morning after night fasting. Blood samples were taken at 10 a.m., including liver functions test, creatinine, serum albumin, Creactive protein (CRP), TT, sex hormone-binding globulin (SHBG), and LH. Laboratory tests were measured by enzymelinked immunosorbent assay (ELISA) (Roche Diagnostics). Calculated free testosterone $(\mathrm{cFt})$ levels were calculated from TT and SHBG and serum albumin with Vermeulen's formula according to mass action laws [11].

\section{Definition}

The American Urological Association [12] defined testosterone deficiency as TT levels below $300 \mathrm{ng} / \mathrm{dL}$ according to testosterone deficiency guidelines, while elevated LH levels more than $9.4 \mathrm{IU} / \mathrm{L}$ was used to define hyper-gonadotropic hypogonadism according to the European Male Aging Study criteria [13].

\section{Statistical analysis}

We conducted statistical analysis by using Statistical Package for the Social Sciences (SPSS), Version 23. All data were checked for suitability for a normal distribution with the Shapiro-Wilk test. All continuous variables were expressed as the mean \pm standard deviation for variables and were compared with independent $t$-test while non-normally distributed expressed as the median and interquartile range (IQR) and were compared using Mann-Whitney test. Categorical variables were expressed as a percentage and Chi-square was carried out to compare between them. Linear regression was used to assess the association of TT as the dependent variable with other statistically significant correlated variables in Pearson correlation (independent variables) in the patients' group. P value less than 0.05 was considered statistically significant.

\section{Results}

A total of 55 recovered, post-hospitalized COVID-19 pneumonia male patients, and 40 age, BMI, and WC-matched participants were enrolled in this study from January 2021 to March 2021. The mean age of the patients was $53.02 \pm 7.385$ years, the prevalence of testosterone deficiency was $50.9 \%$ (28 patients), while the prevalence of hypogonadotropic hypogonadism was $60.7 \%$ (17 patients).

In this study, when we compared clinical and laboratory characteristics between late recovered COVID-19 pneumonia patients and matched participants as the control group, we 
Table 1. Demographic, Clinical, and Laboratory Characteristics of Study Participants

\begin{tabular}{|c|c|c|c|}
\hline & COVID-19 pneumonia patients $(n=55)$ & Matched control $(n=40)$ & P value \\
\hline Age (years) & $53.02 \pm 7.385$ & $51.80 \pm 7.498$ & 0.432 \\
\hline Smoker ratio & $21(38.2 \%)$ & $16(40 \%)$ & 0.858 \\
\hline Hypertension & $29(52.7 \%)$ & $19(47.5 \%)$ & 0.615 \\
\hline Body weight (kg) & $105.93 \pm 15.677$ & $102.73 \pm 16.123$ & 0.334 \\
\hline $\mathrm{WC}(\mathrm{cm})$ & $120.20 \pm 16.875$ & $115.55 \pm 17.255$ & 0.192 \\
\hline CRP (mg/L) & $15.8782 \pm 7.82614$ & $14.175 \pm 7.00417$ & 0.277 \\
\hline $\mathrm{CCI}$ & $3(3)$ & $3(2)$ & 0.128 \\
\hline Albumin $(\mathrm{g} / \mathrm{dL})$ & $4.23 \pm 0.19108$ & $4.1564 \pm 0.27200$ & 0.145 \\
\hline LH (mIU/L) & $7.6836 \pm 2.69298$ & $7.03 \pm 2.47596$ & 0.23 \\
\hline $\mathrm{T} / \mathrm{LH}$ & $0.4731(0.51)$ & $0.6584(0.43)$ & 0.022 \\
\hline Testosterone deficiency prevalence & $28(50.9 \%)$ & $12(30 \%)$ & 0.042 \\
\hline
\end{tabular}

COVID-19: coronavirus disease 2019; ICU: intensive care unit; BMI: body mass index; WC: waist circumference; CCI: Charlson Comorbidity Index; CRP: C-reactive protein; TT: total testosterone; cFT: calculated free testosterone; LH: luteinizing hormone; SHBG: sex hormone binding globulin; T/ $\mathrm{LH}$ : testosterone to $\mathrm{LH}$ ratio.

found statistically significant differences of mean testosterone to $\mathrm{LH}$ ratio, TT, and cFT. Moreover, we found a higher prevalence of testosterone deficiency in the COVID-19 patients group. There were no significant differences between the two groups in mean CCI. Other clinical and laboratory differences between the two groups are detailed in Table 1.

Table 2 demonstrates the comparison of clinical and laboratory parameters between 28 post-COVID-19 testosteronedeficient patients and 27 post-COVID-19 testosterone-sufficient patients. We found significant differences in the means of hospitalization duration, CCI, BMI, WC, and LH. An unexpected result is an insignificant mean difference of CRP between the testosterone-deficient and sufficient patients. Other differences are shown in Table 2.

In this study, Pearson's correlation test was used for exploring any significant relationships between serum TT levels and other parameters. We studied in regression analysis the association between TT as the dependent factor with other significantly correlated parameters as independent variables, hospitalization duration, LH, BMI, WC, and the square root of CCI among 55 COVID-19 patients. We found that WC diameter was the only significant predictor of TT levels. Moreover, after controlling the age, we found that the square root of CCI and WC could significantly predict TT levels as shown in Table 3.

\section{Discussion}

Several studies have highlighted a statistically significant de- crease in testosterone levels with COVID-19 infection; however, the majority of these studies have been conducted in patients with active disease and long-term consequences are still not known and need exploration [14].

In our study, we aimed to evaluate the late impact of COVID-19 pneumonia on androgen levels after recovery. Our main current findings show that recovered COVID-19 pneumonia patients still have lower androgen levels and a higher prevalence of testosterone deficiency in comparison with matched control participants after an average of 60 days. Moreover, visceral obesity was the most significant predictor for low testosterone levels in recovered COVID-19 patients.

The late effect of COVID-19 has been evaluated in rare studies. Our results partially coped with a single-center Chinese study that reported a lower ratio of TT to LH in recovered COVID-19 patients in comparison with age-only matched control and low TT levels were observed only in critically ill patients [15]. On the other hand, our findings were in contrast with another recent Chinese study's findings on recovering COVID-19 patients which did not observe any significant change in comparison with healthy control participants [16]. This contrast may be explained by different patients' selection regarding ethnicity and disease severity.

In the same consent, few studies on hospitalized COVID-19 patients reported generally lower androgen levels with mainly hyper-gonadotropic which pointed to testicular origin. Lower testosterone and higher LH levels in comparison of active COVID-19 patients with age-matched healthy controls were reported $[8,17,18]$. 
Table 2. Comparison of Demographic, Clinical, and Laboratory Characteristics Between COVID-19 Pneumonia Male Patients With Testosterone Deficiency and Patients Without Testosterone Deficiency

\begin{tabular}{llll} 
& Non-deficient testosterone $(\mathbf{n}=\mathbf{2 7})$ & Deficient testosterone $(\mathbf{n}=\mathbf{2 8})$ & P value \\
\hline Age $($ years $)$ & $53.89 \pm 7.617$ & $52.18 \pm 7.191$ & 0.396 \\
Smoker ratio & $10(37 \%)$ & $11(39.3 \%)$ & 0.864 \\
Hospitalization time (days) & $5.19 \pm 1.733$ & $7.46 \pm 3.226$ & 0.002 \\
ICU admission & $3(11.1 \%)$ & $8(28.6 \%)$ & 0.106 \\
Hypertension & $12(44.4 \%)$ & $17(60.7 \%)$ & 0.227 \\
Body weight $(\mathrm{kg})$ & $102.00 \pm 16.502$ & $109.71 \pm 14.108$ & 0.068 \\
Height $(\mathrm{m})$ & $1.7496 \pm 0.05977$ & $1.7339 \pm 0.05750$ & 0.325 \\
BMI $\left(\mathrm{kg} / \mathrm{m}^{2}\right)$ & $33.4579 \pm 5.87564$ & $36.6395 \pm 5.52875$ & 0.043 \\
WC $(\mathrm{cm})$ & $112.93 \pm 16.146$ & $127.21 \pm 14.647$ & 0.001 \\
CRP $(\mathrm{mg} / \mathrm{L})$ & $14.1 \pm 7.90569$ & $17.5929 \pm 7.49004$ & 0.098 \\
CCI & $3(2)$ & $5(4)$ & 0.007 \\
Albumin (g/dL) & $4.2000 \pm 0.26890$ & $4.1143 \pm 0.27314$ & 0.246 \\
SHBG (nmol/L) & $51.2222 \pm 9.86257$ & $46.8750 \pm 9.46348$ & 0.101 \\
cFT (ng/dL) & $7.302593 \pm 1.624783$ & $3.453214 \pm 1.2331$ & 0.0001 \\
LH (mIU/L) & $6.6889 \pm 1.47187$ & $8.6429 \pm 3.23642$ & 0.006 \\
T/LH & $0.2449(0.17)$ & $0.7058(0.29)$ & 0.001 \\
\hline
\end{tabular}

COVID-19: coronavirus disease 2019; ICU: intensive care unit; BMI: body mass index; WC: waist circumference; CCI: Charlson Comorbidity Index; CRP: C-reactive protein; LH: luteinizing hormone; cFT: calculated free testosterone; SHBG: sex hormone binding globulin; T/LH: testosterone to LH ratio.

Similarly, male patients with COVID-19 had lower testosterone levels even in comparison with non-COVID-19 respiratory tract infections and age-matched controls [19]. This may point to a specific pathogenic impact on testicular functions rather than acute phase hypogonadism [20]. Furthermore, testosterone could predict the outcomes of hospitalized COVID-19 patients. Hypotestosteronemia was linked with increased risk in terms of ICU and mortality [19], need of mechanical ventilation or mortality [17], and higher risk of ICU admission and death outcomes [21]. Moreover, testosterone levels were significantly associated with a longer time of hospitalization [19].

However, in contrast to previous studies which pointed to the testicular origin of hypogonadism in active COVID-19 patients, a recent finding from a large case-control study [21] reported a very high prevalence of testosterone deficiency around 90\%. However, in contrast with other studies, hypogonadism patients were predominately hypogonadotropic hypogonadism pointing to hypothalamic and/or pituitary origin. Hypogonadism patients in our study were predominately hypogonadotropic suggestive of hypothalamic or pituitary origin.

Few pathogenic mechanisms can explain testosterone deficiency in COVID-19 pneumonia. Acute stage hypogonadism in male COVID-19 patients like other critical infections may be explained via the mechanism of secondary immune reactions and oxidant sensitive-inflammatory pathways. The SARS$\mathrm{CoV}-2$ infection could induce secondary inflammation and oxidative stress is more likely to impair androgen synthesis [22], yet, our patients in this study were recovered with average 60 day period. Another mechanism could be via prolactin levels change. Few studies reported abnormally elevated prolactin

Table 3. Linear Regression Models Predicting Total Testosterone Levels in Recovered, Post-Hospitalized for COVID-19 Pneumonia Male Patients

\begin{tabular}{|c|c|c|c|c|c|c|}
\hline & \multicolumn{3}{|c|}{ Model 1} & \multicolumn{3}{|c|}{ Model 2} \\
\hline & B & $\beta$ & $P$ value & B & $\beta$ & P value \\
\hline WC & -5.895 & -2.932 & 0.005 & -5.941 & -2.991 & 0.004 \\
\hline $\mathrm{LH}$ & -3.154 & -0.470 & 0.641 & -1.600 & -0.238 & 0.813 \\
\hline Hospitalization time & -9.768 & -1.599 & 0.116 & -9.866 & -1.635 & 0.109 \\
\hline
\end{tabular}

Model 1 was unadjusted. Model 2 adjusted for age. COVID-19: coronavirus disease 2019; WC: waist circumference; BMI: body mass index; LH: luteinizing hormone; Sqrt $\mathrm{CCl}$ : square root of Charlson Comorbidity Index. 
levels in COVID-19 pneumonia which has a known suppressive effect on pituitary gonadotropin secretion although hyperprolactinemia might be attributed to other etiologies like drugs and stress [19, 23, 24]. Furthermore, direct damage of testicular tissue by the SARS-CoV-2 virus was hypothesized. High expression of ACE2 receptors in Leydig and Sertoli cells could suggest direct SARS-CoV-2 impact at the testicular level in active COVID-19 patients; however, the extent of this damage still needs to be verified. There are recent studies that reported injury to Sertoli cells and seminiferous tubules, Leydig cells loss in COVID-19 patients [25], and damage of seminal tubules in post mortem COVID-19 patients [26]. Another mechanism via the ACE2 pathway was postulated. The high expression of ACE2 receptors at Leydig cells and its decreased expression by SARS-CoV-2 leads to subsequently enhanced angiotensin II levels [27]. Angiotensin II could reduce both basal and LHstimulated testosterone synthesis by Leydig cells [7]. Another mechanism could be postulated by the obesity effect. Obesity is one of the risk factors for severity of COVID-19 disease and connects with COVID-19 by few mechanisms as immune system activity attenuation and chronic inflammation [28]. At the same time, obesity particularly visceral obesity is a wellknown risk factor for testosterone deficiency in males [29]. Similarly, in our study result, visceral obesity was the most significant predictor for low testosterone levels in recovered COVID patients. Hence, obesity is considered a risk factor for both COVID-19 and testosterone deficiency, therefore, visceral obesity may be the main link between COVID-19 and hypogonadism. Another mechanism that could postulate testosterone deficiency in COVID-19 pneumonia is corticosteroids. Corticosteroids likely are involved in testosterone deficiency in COVID-19 disease. The use of corticosteroids and stressinduced hypercortisolism might have a suppressive impact on the hypothalamus-pituitary-gonadal axis. Cortisol can disrupt and suppress the hypothalamus-pituitary-gonadal axis. Corticosteroid could suppress gonadotropin-releasing hormone at the hypothalamic level and could affect the pituitary gonadotropin action at the testicular level [30]. We excluded patients with corticosteroid use in the last 4 weeks to avoid cortisol disruption on hypothalamus-pituitary-gonadal axis. Lastly, a recent hypothesis of the production of anti-sperm antibodies was postulated. Following damage to the blood-testicular barrier, anti-sperm antibodies were postulated to be produced and involved in hypogonadism pathogenesis [31].

To the best of our knowledge, despite this article does not present first the evaluation of reproductive hormonal levels in patients with COVID-19, our study for the first time demonstrated lower testosterone levels in hospitalized patients with COVID-19 after recovery. Our study has some points of strength. Firstly, it evaluates the late effect of the SARSCoV-2 virus on testosterone levels after recovery. Secondly, our participants as control were matched according to age, and anthropometric parameters such as WC and BMI, which are known to affect testosterone levels by a large number of studies. Thirdly, we excluded any history of disorders known to affect androgen levels even DM. Lastly, we evaluated both free testosterone and TT levels.

The current study is certainly not devoid of limitations, which include relatively smaller sample size, single center- based study, and the study could not evaluate these COVID-19 patients prospectively. Another limitation is that we did not evaluate follicle-stimulating hormone (FSH) and prolactin due to limited funding for this research as this study was funded by the authors themselves. Another limitation is that a pre-existing testosterone deficiency could not be excluded in all our participants although few patients have normal testosterone report with variable time, yet, we excluded all disease which could affect androgen level and set our inclusion criteria for age between 18 and 65 for avoiding known impact of age on androgen level and match our control group. Lastly, we recruited hospitalized patients only. Hence the effect of mild COVID-19 was not evaluated even our control participants may have asymptomatic COVID-19 and our results could be applied to moderate and severe cases of COVID-19 only.

In this study, we highlighted the need for gonadal function evaluation among patients recovered from SARS-CoV-2 infection particularly severe and critical patients, and further studies are invited to verify our study findings.

\section{Conclusion}

Late recovered post-hospitalized COVID-19 pneumonia patients still have lower androgen levels and a higher prevalence of testosterone deficiency in comparison with matched control participants. Moreover, visceral obesity was the most significant predictor of low testosterone levels in recovered COVID-19 patients. These results need more confirmation in other communities and more follow-up studies.

\section{Acknowledgments}

This work was supported by Specialized Medical Hospital staff at Mansoura University.

\section{Financial Disclosure}

None to declare.

\section{Conflict of Interest}

None to declare.

\section{Informed Consent}

Written informed consent was obtained from each participant.

\section{Author Contributions}

MA and AA contributed to the conception and interpretation of the data. AN and ES performed the laboratory tests. MA performed the statistical analysis. MA and AA were involved 
in writing, reviewing, and editing the manuscript. All authors read and approved the final manuscript.

\section{Data Availability}

The data supporting the findings of this study are available from the corresponding author upon reasonable request.

\section{References}

1. Gralinski LE, Menachery VD. Return of the coronavirus: 2019-nCoV. Viruses. 2020;12(2):135.

2. Isidori AM, Pofi R, Hasenmajer V, Lenzi A, Pivonello R. Use of glucocorticoids in patients with adrenal insufficiency and COVID-19 infection. Lancet Diabetes Endocrinol. 2020;8(6):472-473.

3. Peckham H, de Gruijter NM, Raine C, Radziszewska A, Ciurtin C, Wedderburn LR, Rosser EC, et al. Male sex identified by global COVID-19 meta-analysis as a risk factor for death and ITU admission. Nat Commun. 2020;11(1):6317.

4. Mohamed MS, Moulin TC, Schioth HB. Sex differences in COVID-19: the role of androgens in disease severity and progression. Endocrine. 2021;71(1):3-8.

5. Gheblawi M, Wang K, Viveiros A, Nguyen Q, Zhong JC, Turner AJ, Raizada MK, et al. Angiotensin-converting enzyme 2: SARS-CoV-2 receptor and regulator of the reninangiotensin system: celebrating the 20th anniversary of the discovery of ACE2. Circ Res. 2020;126(10):1456-1474.

6. Wang $\mathrm{Z}, \mathrm{Xu} \mathrm{X}$. scRNA-seq profiling of human testes reveals the presence of the ACE2 receptor, a target for SARS-CoV-2 infection in spermatogonia, Leydig and Sertoli cells. Cells. 2020;9(4):920.

7. Douglas GC, O'Bryan MK, Hedger MP, Lee DK, Yarski MA, Smith AI, Lew RA. The novel angiotensin-converting enzyme (ACE) homolog, ACE2, is selectively expressed by adult Leydig cells of the testis. Endocrinology. 2004;145(10):4703-4711.

8. Okcelik S. COVID-19 pneumonia causes lower testosterone levels. Andrologia. 2021;53(1):e13909.

9. Salonia A, Pontillo M, Capogrosso P, Gregori S, Tassara M, Boeri L, Carenzi C, et al. Severely low testosterone in males with COVID-19: A case-control study. Andrology. 2021.

10. Charlson ME, Pompei P, Ales KL, MacKenzie CR. A new method of classifying prognostic comorbidity in longitudinal studies: development and validation. J Chronic Dis. 1987;40(5):373-383.

11. Vermeulen A, Verdonck L, Kaufman JM. A critical evaluation of simple methods for the estimation of free testosterone in serum. J Clin Endocrinol Metab. 1999;84(10):36663672.

12. Mulhall JP, Trost LW, Brannigan RE, Kurtz EG, Redmon JB, Chiles KA, Lightner DJ, et al. Evaluation and management of testosterone deficiency: AUA guideline. J Urol. 2018;200(2):423-432.

13. Tajar A, Forti G, O'Neill TW, Lee DM, Silman AJ, Finn
JD, Bartfai G, et al. Characteristics of secondary, primary, and compensated hypogonadism in aging men: evidence from the European Male Ageing Study. J Clin Endocrinol Metab. 2010;95(4):1810-1818.

14. Delle Fave RF, Polisini G, Giglioni G, Parlavecchio A, Dell'Atti L, Galosi AB. COVID-19 and male fertility: Taking stock of one year after the outbreak began. Arch Ital Urol Androl. 2021;93(1):115-119.

15. Ma L, Xie W, Li D, et al. Effect of SARS-CoV-2 infection upon male gonadal function: A single centerbased study. Medxriv. 2020.

16. Xu H, Wang Z, Feng C, Yu W, Chen Y, Zeng X, Liu C. Effects of SARS-CoV-2 infection on male sex-related hormones in recovering patients. Andrology. 2021;9(1):107114.

17. Rastrelli G, Di Stasi V, Inglese F, Beccaria M, Garuti M, Di Costanzo D, Spreafico F, et al. Low testosterone levels predict clinical adverse outcomes in SARS-CoV-2 pneumonia patients. Andrology. 2021;9(1):88-98.

18. Schroeder M, Tuku B, Jarczak D, et al. The majority of male patients with COVID-19 present low testosterone levels on admission to intensive care in Hamburg, Germany: a retrospective cohort study. medRxiv. 2020.

19. Kadihasanoglu M, Aktas S, Yardimci E, Aral H, Kadioglu A. SARS-CoV-2 Pneumonia Affects Male Reproductive Hormone Levels: A Prospective, Cohort Study. J Sex Med. 2021;18(2):256-264.

20. Ma L, Xie W, Li D, Shi L, Ye G, Mao Y, Xiong Y, et al. Evaluation of sex-related hormones and semen characteristics in reproductive-aged male COVID-19 patients. J Med Virol. 2021;93(1):456-462.

21. Cayan S, Uguz M, Saylam B, Akbay E. Effect of serum total testosterone and its relationship with other laboratory parameters on the prognosis of coronavirus disease 2019 (COVID-19) in SARS-CoV-2 infected male patients: a cohort study. Aging Male. 2020;23(5):1493-1503.

22. Sengupta P, Dutta S. COVID-19 and hypogonadism: secondary immune responses rule-over endocrine mechanisms. Hum Fertil (Camb). 2021:1-6.

23. Sen A. Repurposing prolactin as a promising immunomodulator for the treatment of COVID-19: Are common Antiemetics the wonder drug to fight coronavirus? Med Hypotheses. 2020;144:110208.

24. Garg MK, Gopalakrishnan M, Yadav P, Misra S. Endocrine involvement in COVID-19: mechanisms, clinical features, and implications for care. Indian J Endocrinol Metab. 2020;24(5):381-386.

25. Haghpanah A, Masjedi F, Alborzi S, Hosseinpour A, Dehghani A, Malekmakan L, Roozbeh J. Potential mechanisms of SARS-CoV-2 action on male gonadal function and fertility: Current status and future prospects. Andrologia. 2021;53(1):e13883.

26. Yang M, Chen S, Huang B, Zhong JM, Su H, Chen YJ, Cao Q, et al. Pathological findings in the testes of COVID-19 patients: clinical implications. Eur Urol Focus. 2020;6(5):1124-1129.

27. Shen Q, Xiao X, Aierken A, Yue W, Wu X, Liao M, Hua J. The ACE2 expression in Sertoli cells and germ cells may cause male reproductive disorder after SARS-CoV-2 
infection. J Cell Mol Med. 2020;24(16):9472-9477.

28. Petrakis D, Margina D, Tsarouhas K, Tekos F, Stan M, Nikitovic D, Kouretas D, et al. Obesity a risk factor for increased COVID19 prevalence, severity and lethality (Review). Mol Med Rep. 2020;22(1):9-19.

29. Fui MN, Dupuis P, Grossmann M. Lowered testosterone in male obesity: mechanisms, morbidity and manage- ment. Asian J Androl. 2014;16(2):223-231.

30. Whirledge S, Cidlowski JA. Glucocorticoids, stress, and fertility. Minerva Endocrinol. 2010;35(2):109-125.

31. Fan C, Lu W, Li K, Ding Y, Wang J. ACE2 expression in kidney and testis may cause kidney and testis infection in COVID-19 patients. Front Med (Lausanne). 2020;7:563893. 